\title{
Metastatic Triple Negative Breast Cancer: The New Era of Thinking
}

Amrallah A. Mohammed*1,2, Mohamed A. Elbassuiony², Hanaa Rashied ${ }^{3}$

${ }^{1}$ Medical Oncology Department, Faculty of Medicine, Zagazig University, Egypt

${ }^{2}$ King Salman Armed Forces Hospital, Tabuk City

${ }^{3}$ El-mabra Hospital, Zagazig, Egypt

Received 14 December 2018; Accepted 26 April 2019

\begin{abstract}
The heterogeneity of triple negative breast cancer (TNBC) is reflected in a bizarre response to therapy. Although it is chemotherapy sensitive, the failure is the usual pathway either in local or distance status. With progression in Gene Expression Profile (GEP) and other molecular techniques, TNBC is divided into sub-types with unique pathways. In the current review, we are trying to highlight based on the molecular classification of TNBC and the management based on every type.
\end{abstract}

Keywords: Breast cancer • triple-negative breast cancer • subtypes of TNBC

\section{Introduction}

Breast cancer $(\mathrm{BC})$ is often referred to as a heterogeneous disease, including different subtypes with a specific clinicopathological entity and biological attitude, affecting treatment options and survival outcome.

$\mathrm{BC}$ diagnosis is a morphological micro invasion, immunohistochemistry staining $(\mathrm{IHC})$, and molecular analysis.

Although histological evaluation and routine IHC are essential, they did not explain the difference in disease behavior.

After the gene expression profile and DNA microarrays, Perou et al. proposed that BC could be classified into five subtypes and a normal breast-like ${ }^{[1]}$. After that, various subtypes have been suggested. During normal mammary gland development, the stem cell will pass through different stages from the luminal progenitor, late luminal progenitor then differentiated luminal cells. The molecular classification of $\mathrm{BC}$ and the relation between breast cell development and the corresponding molecular types is illustrated in Figure 1 and 2.

The pathological report of $\mathrm{BC}$ must include at least a comment on the status of estrogen (ER), progesterone (PR) receptors, the TNM (tumor-node-metastasis) classification, and HER-2 status either by IHC or PCR ${ }^{[2]}$.

According to the American Society of Clinical Oncology/College of American Pathologists (ASCO/ $\mathrm{CAP}$ ), triple negative breast cancer (TNBC) defined as testing negative for ER, PR and HER-2 over-expression/ amplification, resulting in less probability to respond to anti-HER-2 or some hormonal therapies ${ }^{[3]}$.

Despite, TNBC is characterized by a high rate of relapse, visceral metastasis and poor survival; most have an excellent chemo-sensitivity without specific protocols $^{[4]}$.

Early, TNBC and basal cell types were described as one entity due to matching many analogies, even though, 
they were used interchangeably. In a study conducted by Prat et al., on 412 TNBC and 473 basal cell type $\mathrm{BC}$ revealed that $78.6 \%$ of TNBC was a basal type, and $68.5 \%$ of basal type was TN. They are two subtypes of $\mathrm{BC}$ with distinct features ${ }^{[5]}$.

TNBC accounts for $15 \%$ to $17 \%$ of $B C$, mainly affecting the premenopausal of African and Hispanic descent. Most of them have a high proliferative index and high pathological characters. Although up to $22 \%$ of TNBC initially respond to chemotherapy, local recurrence and distance metastasis peak were mainly in the first three years of therapy ${ }^{[6]}$. In a study conducted by Lin et al., on 116 patients with TNBC at Dana-Farber, Cancer Institute, revealed short disease-free interval (DFS), increase in visceral metastasis $(74 \%)$ with central nervous system metastasis in up to $46 \%$ of patients ${ }^{[7]}$.

This aim of this review is to identify the molecular subtypes of TNBC and the affect treatment selection.

\section{The diversity of histological features and the survival outcome}

Although many TNBC subtypes are of high-grade invasive duct carcinomas, a small subset has different pathologic patterns and indolent clinical course as adenoid cystic carcinoma, metaplastic carcinoma, and medullary carcinomas, each type has distinct histologypathological features.

With the discrepancy in therapy type and duration, TNBC subtypes had shown the variable pattern in both relapse-free survival (RFS) and distance metastasisfree survival (DMFS), with luminal androgen receptor subtype, had a significantly worse prognosis alongside basal-like, immune modulatory and mesenchymal subtypes $(p<0.005)$. Owing to this, the investigators are thinking about biologic subtypes that could be evaluated by gene expression profile reflecting the diversity of clinical behavior ${ }^{[8]}$.

\section{Molecular classifications of TNBC}

In 2011, Lehmann et al. identified six unique TNBC subtypes of Gene Expression Profile (GEP) analysis of 587 TNBC cases derived from 21 BC data set. It included: two basal-like subtypes (BL1 and BL2); an immune modulatory (IM) subtype; two mesenchymal subtypes (M and MSL), and a luminal androgen receptor (LAR) subtype, called Vanderbilt classification ${ }^{[9]}$.

PAM50 is a 50 -gene qPCR assay recognized five intrinsic TNBC subtypes encompassing: basal-like, HER2-enriched, normal-like, luminal $B$, and luminal $A^{[10]}$.
Over the years, other molecular classifications for TNBC had emerged. The Baylor subtype: in a single institute study in the Baylor University, Burstein et al., in 2014 evaluated 198 TNBC tumor samples by mRNA expression and DNA profiling. They classified TNBC into four subtypes: basal-like immunosuppressed, basal-like immune-activated, mesenchymal, and LAR subtype ${ }^{[11]}$. In 2015, another classification subtype of TNBC was proposed by investigators in a single center in France. Owing to GEP for 194 TNBC, they identified three subtypes: LAR, basal-like with a low immune response and high M2-like macrophages, basal-enriched with a high immune response and low M2-like macrophages, which are referred as French subtype ${ }^{[12]}$.

\section{Main subtypes of TNBC and directed therapy}

\subsection{Basal-like subtype}

It was called basal-like due to the distinctive expression of CK 5, 6, or 17, which are basal type markers ${ }^{[13]}$. t represented about $80 \%-90 \%$ of $\mathrm{BC}$ with a germline mutation in BRCA (gBRCAm) and in $60 \%-72 \%$ of TNBC $^{[14]}$.

The biological pathways involving the cell division and DNA damage response leading to speeding up cell proliferation. The basal-like subtype relates to mutations in RB and p53 in addition to gBRCAm, which may explain the high aneuploidy observed in these subtypes ${ }^{[15,16]}$.

As we know, BRCA 1/2 are tumor suppressor genes involved with poly ADP-ribose polymerase (PARP) enzymes in repairing damaged DNA. Therefore, medications as PARP inhibitors (PARPi) and platinum agents have a considerable role in gBRCAm. BRCAness is referred to tumors that have the molecular characters of BRCA mutant tumors. The homologous recombination deficiency (HRD) was used as biomarkers that identify BRCAness tumors ${ }^{[17]}$.

\subsection{PARP inhibitors}

On January 12, 2018, olaparib, a PARPi, was approved by the Food and Drug Administration (FDA) for patients with HER2-negative metastatic breast cancer (MBC) with $\mathrm{g}$ BRCA $1 / 2 \mathrm{~m}$ post-treatment with at least two lines of chemotherapy either in the adjuvant, neoadjuvant, or metastatic setting. The approval based on the results of OlympiAD trial; randomized, open-label multicenter phase III study that included 205 patients treated with olaparib $300 \mathrm{mg}$ BID in one arm, and the other arm 
included 97 patients treated with standard of care (SOC) chemotherapy including (capecitabine; eribulin mesylate and vinorelbine) in a 21-day cycle. Eligibility criteria included MBC HER2-negative, with gBRCAm (either suspected or confirmed); TNBC: after two or more lines of chemotherapy or hormone receptors positive (HR-positive): progressed on $\geq 1$ endocrine therapy or unsuitable for it. The primary endpoint was progression-free survival (PFS) assessed by a blinded independent central review (BICR). Secondary endpoints were time to second progression/death, overall survival (OS), overall response rate (ORR), safety, health-related quality of life (HRQoL). The results revealed that median PFS was 7.0 and 4.2 months (HR: 0.58 95\% Cl: 0.43-0.80; $\mathrm{P}<.001$ ), in the olaparib and chemotherapy arms, respectively. Moreover, there was no statistical significance as regards the median OS; 19.3 and 19.6 months (HR: 0.90 (95\% Cl: 0.63-1.29; P $=.57$ ), respectively ${ }^{[18]}$.

Nausea, anemia, vomiting, and fatigue were represented the most common adverse effects (AEs). Patient's identifications for olaparib use in MBC based on BRACAnalysis CDx® test (Myriad Genetic Laboratories, Inc.) to detect gBRCAm, which is FDA approved.

$A B R A Z O$ is a phase II trial, evaluating another PARPi; talazoparib in gBRCAm MBC, including two cohorts; cohort 1 with 48 patients with previous response to platinum-based treatment, a progressive disease more than 8 weeks after the last cycle. Cohort 2 involved 35 patients, received at least 3 cycles of chemotherapy without prior platinum-containing regimens. The primary results showed promising survival outcome especially in platinum naïve ${ }^{[19]}$.

EMBRACA is another ongoing phase III comparing talazoparib versus physician's choice of chemotherapy in gBRCA1/2+ MBC. The primary endpoint was PFS and secondary endpoints were: ORR, OS, safety, DoR (duration of response), QoL (quality of life) ClinicalTrials. gov. NCT01945775.

\subsection{Platinum}

The benefit from platinum compounds, especially carboplatin, had been evaluated in the neoadjuvant setting in TNBC. The pathologic complete response (pCR) was ranged from $54 \%$ to $58.7 \%$ versus $40 \%$ to $37.5 \%$ in carboplatin versus non carboplatin arm, respectively ${ }^{[20,21]}$.

In the CBCSG006 study, Hu et al. concluded that the combined cisplatin/gemcitabine may be a preferred first-line or alternative option compared with paclitaxel/ gemcitabine $\mathrm{mTNBC}^{[22]}$.
Moreover, phase II tnAcity trial, compared nabPaclitaxel plus carboplatin or gemcitabine versus gemcitabine plus carboplatin as first-line therapy of patients with $\mathrm{mTNBC}$. Randomization was 1:1:1 with Nab-paclitaxel $125 \mathrm{mg} / \mathrm{m} 2$ + carboplatin AUC 2, Nabpaclitaxel $125 \mathrm{mg} / \mathrm{m} 2$ + gemcitabine $1000 \mathrm{mg} / \mathrm{m} 2$ or gemcitabine $1000 \mathrm{mg} / \mathrm{m} 2$ + carboplatinAUC 2, all on Days 1, 8 of 21-day cycle. The outcome results demonstrated that Nab-paclitaxel/carboplatin had a significant PFS in comparison to the other combinations ${ }^{[23]}$.

The triple negative trial (TNT), is another phase III trial, including 376 patients with mTNBC or gBRCA1/2m, comparing carboplatin (AUC6 Q3W x 6 cycles: $n=188$ ) to docetaxel $(100 \mathrm{mg} / \mathrm{m} 2 \mathrm{Q} 3 \mathrm{~W} \times 6$ cycles: $\mathrm{n}=188)$. The primary endpoint was ORR in intent to treat (ITT) was statistically significantly higher in carboplatin arm across gBRCA1/2m, p-value $=0.03^{[24]}$.

\subsection{Luminal AR subtype}

It is the most distinguished subtype, accounting about $11 \%$ of TNBC characterized by higher significant levels of AR expression. It represented the backbone for targeting with anti-androgen agents. Due to the similarity between AR, ER, and PR, CAP/ASCO guidelines used the same IHC scoring method. The cut off value used was $1 \%$ in some studies and $10 \%$ in others ${ }^{[25,26]}$.

Extrapolation from the anti-androgen effect of bicalutamide on the prostate carcinoma, Gucalp et al. evaluated the clinical benefit of it in LAR subtype. The clinical benefit rate (CBR) was 19\% in AR-positive patients, which boost believe that AR blockade may be an option in this subtype ${ }^{[27]}$.

Another, multi-center open-label, single-arm phase II trial evaluating enzalutamide in 75 patients with ARpositive $\mathrm{mTNBC}$ presented by Traina $\mathrm{T}$ et al. in the annual meeting of the ASCO 2017, reported that CBR at 16 and 24 weeks were $35 \%$ and $29 \%$ with enzalutamide dose of $160 \mathrm{mg}$ daily[28].

\subsection{Immune modulatory subtype}

The IM subtype, mentioned in the three molecular subtypes; Vanderbilt, Baylor, and French classification, is characterized by the enriching of genes involved in immune pathways. Consequently, the IM subtype may benefit from immune checkpoint inhibitors (ICls).

Recently, international, multi-cohort phase II trial (KEYNOTE-086), presented in ASCO 2017 evaluated pembrolizumab (200 mg iv/3 weeks until disease progression or unaccepted toxicity) in programmed 
death-ligand 1 positive (PD-L1-positive), mTNBC patients. The selected patients were divided into two cohorts: cohort A encompassed post one or more systemic therapy, and cohort B included previously untreated patients.

The primary endpoint was ORR and secondary endpoints were DoR, DCR, PFS, and OS. After a median follows up (10.9 months for cohort $A ; 7.0$ months for cohort B), ORR and DCR were better in cohort $B$ (naïve patients) than in cohort A, either in PD-L1 positive or PD-L1 negative $(23 \%, 4.8 \%, 4.7 \%$ and $23 \%, 9.5 \%$, $4.7 \%$, respectively). The poor prognostic features included: $\mathrm{LDH}>\mathrm{ULN} \geq 3$ metastatic organ sites, liver metastases, and visceral disease were numerically associated with lower ORR. The safety evaluation was accepted; treatment-related and immune-related AEs were $4.1 \%$ and $1.2 \%$ in patients resulting in treatment discontinuation without death related $\mathrm{AEs}^{[29]}$.

The use of ICls combined with radiotherapy and/ or chemotherapy (especially in metronomic manner) is considered a relatively new avenue. There are at least two mechanisms explaining the rationale of this combination. Firstly, the chemotherapy is roughening up the surface of cancer, which enables the immune system to better recognize and fight cancer as a foreign object. Secondary, radiation-induced cancer cell damage promotes the priming and activation of cytotoxic T cells and improves the recruitment and infiltration of immune cells. This synergistic effect makes the combination a fertile land for more researchers ${ }^{[30]}$.

The TONIC trial is an adaptive, randomized, noncomparative phase II study that evaluated the nivolumab after induction treatment with radiotherapy or low dose chemotherapy in $\mathrm{mTNBC}$. The eligibility criteria were patients after at least 3 lines or more of palliative chemotherapy, after a median follow-up of 10.8 months, ORR for the entire cohort was $22 \%$ and the median PFS was 3.4 months. The investigators reported that the priming with either irradiation or chemotherapy leading to roughening up of the surface of cancer followed by ICPI was a promising option in TTNBC $^{[31]}$.

Based on the findings of the IMpassion130 trial, the FDA granted atezolizumab (PD-L1 inhibitor) combo priority review for frontline $\mathrm{MTNBC}$ with PD-L1-positive on Nov 13, 2018.

To briefly outline, the IMpassion130 is a doubleblind phase III trial that evaluated the combination of nab-paclitaxel at $100 \mathrm{mg} / \mathrm{m} 2$ plus atezolizumab at $840 \mathrm{mg}$ versus nab-paclitaxel alone in patients with naïve metastatic or locally advanced TNBC. Treatment was continued until unacceptable toxicity or disease progression. There was a benefit in both PFS and OS in the PD-L1-positive patients with intratumoral positivity of CD8+ T-cells, sTILs, or BRCA1/2 in atezolizumab group, but no benefit is evident in the PD-L1-negative subset.

The discontinuation of therapy due to AEs was $15.9 \%$ of the patients in the combined group compared to $8.2 \%$ for patients in the chemotherapy group only. The safety was comparable with the neutropenia represented the common grade 3/4 AEs. Despite that the absolute benefit of the IMpassion130 is small (2.5-month benefit, from 5.0 months with placebo to 7.5 months with atezolizumab; $\mathrm{P}<.0001$ ), it changed the treatment landscape in $\mathrm{mTNBC}$ and offers definitive benefit from immunotherapy in a subset of patients for which the chemotherapy was the only option ${ }^{[32]}$.

\section{Mesenchymal subtype}

It is characterized by up-regulation of pathways enriched in cell motility, epithelial-to-mesenchymal transition (EMT), cell differentiation, extracellular matrix receptor interaction, and growth factor signaling pathways (Wnt pathway, anaplastic lymphoma kinase[ALK] pathway, and PI3K/AKT/mTOR pathway); in addition, there were data that anticipated the implication of NOTCH pathway, making the probability of targeting these pathways a feasible option ${ }^{[33]}$.

Eribulin, microtubule inhibitors with its significant suppressor effect on EMT pathway, may be actionable in ML subtype. Moreover, the fibroblast growth factor receptor (FGFR) inhibitors could be used in targeting this pathway ${ }^{[34-36]}$.

\section{Discussion}

TNBC is a heterogeneous disease characterized by higher rates of relapse and rapid progression. Chemotherapy is the standard of care of which most of the patients quickly develop treatment failure. In the metastatic stage, 5 -year survival rate is $22 \%$ compared with $27 \%$ in non-TNBC ${ }^{[37]}$.

Historically, the systemic hormonal treatment of BC started in 1971 and it depended on hormonal receptor status. Trastuzumab was approved for treatment in HER-2 positive on September 25, 1998. Among the progress in the molecular field, Perou et al. and Sorlie et al. proposed intrinsic molecular subtypes in 2000-2001. Lehmann et al. worked on the molecular classification of TNBC from 2011 till refinement (TNBC type-4) in $2016^{[38]}$.

Due to the lack of the specific targeted agents either anti-HER-2 or hormonal treatment, the chemotherapy 
is still the cornerstone in the management, despite the associated side effects. Figure (3) illustrated the main available chemotherapeutic groups.

However, at the genomic level, TNBC has at least four different subtypes with specific biological pathways, each reflecting its heterogeneity, which is represented by the difference in chemotherapy sensitivity, time of recurrence, and type of metastasis.

Understanding the molecular basis and the principal driver's mutation will allow the researchers to select effective therapy and maximize the therapeutic outcome. Figure (4) showed TNBC subtype pathways, and possible targeting drugs.

Olaparib, PARPi, is the only approved nonchemotherapy agent used in the management of mTNBC with gBRCAm with statistically significant improvement in PFS and acceptable toxicity profile.

Despite the overexpression of EGFR in basal-like tumors, cetuximab, an anti-EGFR monoclonal antibody had failed to improve the survival outcome when added to carboplatin/irinotecan in pretreated TNBC in TBCRC 001 study, most likely due to alternative pathway's activations $^{[34]}$.

With the new era of immunotherapy and their approval in many types of cancer as NSCLC, and colon cancer with the promising results in the de novo diagnosed mTNBC, we are expecting the approval of many immunotherapy agents soon.

There is growing evidence of the relationship between tumor mutation burdens (TMB); tumor infiltrating lymphocyte (TIL) and response to ICls, which may be used as biomarkers to select the immunotherapy, similar to mismatch repair deficiency ${ }^{[39]}$.

In the survival analysis of IMpassion130 trial referring to PD-L1 expression and CD8 expression, TIL or BRCA1/2 mutation status, it becomes apparent that patients positive for CD8, TIL, or BRCA1/2 mutations benefited from immunotherapy (i.e., the addition of atezolizumab) only when collectively positive for PD-L1. So, it is better to test PD-L1 expression as a routine to identify patients who would most probably benefit from ICls. Moreover, the efforts are now directed to change the tumor from an immunological cold state (low TMB) to behave as hot tumors (high TMB), which can be done by both anti-PD-1 therapy with other agents ${ }^{[29]}$.

Meanwhile, although the tumor progression had been for the activation of both EMT and MET pathway, the reported results from c-MET-directed modalities are not satisfied in metastatic unselected TNBC ${ }^{[40]}$.

Notably, Hon et al. developed a proposal to classify the TNBC by AR status into AR +ve or AR -ve, the latter called quadruple negative breast cancer (QNBC). QNBC is not widely used in our daily clinical practice, it is characterized by lacking for ER, PR, HER-2, and AR making the target therapies that are not achievable. However, recent expressions of unique proteins have been identified in QNBC and may be amenable to direct therapy. Fatty acyl-CoA synthetase 4 (ACSL4) and S-phase kinase-associated protein 2 (SKP2) are examples of proteins that are under evaluation as a biomarker and targetable in QNBC ${ }^{[41]}$.

In general, the response of LAR subtype to chemotherapy is less against other subtypes. The link between the lower Ki-67 index and AR positivity may explain these phenomena. In addition, although, the AR-V7 proposed to be associated with poor response to androgen ablation therapy and poor prognosis in prostate cancer, its role in TNBC is still questionable ${ }^{[42]}$.

HER-2 enriched is a unique subtype with a low level of HER-2 overexpression, which was not included in the Lehmann proposal. However, Perou et al. identified a type similar to the Her-2 overexpression type in TNBC, through GEP ${ }^{[1]}$. Astonishing, the analysis of the NSABP-B31 trial revealed the achievement of $C B$ in some cases with HER-2 negative $B C$ when treated with trastuzumab. A similar result was obtained from a phase II trial evaluating the use of HER2 vaccine AE $37^{[43,44]}$.Actually, more efforts are needed to understand the mechanisms and value of anti-Her-2 therapy in TNBC.

At this moment, many targets and biological therapies are under investigations to treat different TNBC subtypes based on mutation drivers. The most important clinical trials in $\mathrm{mTNBC}$ are summarized in Table 1.

Although we have meaningful progress in the molecular classification of TNBC based on GEP, it is still experimental. The presence of plenty of gene's involvement represents the main obstacle in clinical application.

It is imperative to remain incorporating GEP into clinical studies of targeted therapies in TNBC, to personalize treatment, and consequently, obtain a more effective result.

\section{Conclusion}

TNBC is a heterogeneous disease with different molecular pathways, which will soon affect therapy selection. The exact subtypes of TNBC are an open area of discussion. Unfortunately, most of the clinical studies on TNBC had enrolled unselected patients, which leads to an attenuation of drug output. We wish to change the landscape for TNBC treatment in the near futures, through the introduction of new and more effective 
biological therapy, applying for precision medicine in clinical practice in diagnosing, treatment and prevention of TNBC to get actual progress.

\section{References}

[1] Perou CM, Sørlie T, Eisen MB, van de Rijn M, Jeffrey SS, Rees CA, et al. Molecular portrait of human breast tumors. Nature. 2000; 406:747-752.

[2] [Guideline] NCCN Clinical Practice Guidelines in Oncology: Breast Cancer. V 1.2016. National Comprehensive Cancer Network. Available at http://www.nccn.org/professionals/physician_gls/ pdf/breast.pdf. Version 3.2018 - October 25, 2018; Accessed: December 7, 2018.

[3] Wolff AC, Hammond ME, Hicks DG, Dowsett M, McShane LM, Allison $\mathrm{KH}$, et al. Recommendations for human epidermal growth factor receptor 2 testing in breast cancer: American Society of Clinical Oncology/College of American Pathologists clinical practice guideline update. J. Clin.Oncol.2013: 31, 3997-4013.

[4] Portha $H$, Jankowski C, Cortet M, Desmoulins I, Martin E, Lorgis V, Arnould L, Coutant C. [Nonmetastatic triple-negative breast cancer in 2016: Definitions and management]. Gynecol Obstet Fertil. 2016 Sep;44(9):492-504.

[5] Prat A, Adamo B, Cheang MC. Molecular characterization of basal-like and non-basal-like triple-negative breast cancer. Oncologist. 2013; 18:123-33.

[6] Chandra D, Suresh P, Sinha R, Azam S, Batra U, Talwar V, Kumar K, Mehta A.Eight Year Survival Analysis of Patients with Triple Negative Breast Cancer in India. Asian Pac J Cancer Prev. 2016; 17:2995-9.

[7] Jia Jin, Yu Gao, Jian Zhang, Leiping Wang, Biyun Wang, Jun Cao, Zhimin Shao, Zhonghua Wang.Incidence, pattern and prognosis of brain metastases in patients with metastatic triple negative breast cancer. BMC Cancer. 2018; 18: 446.

[8] Weigelt B, Ng CK, Shen R, Popova T, Schizas M, Natrajan R, et al. Metastatic breast carcinomas display genomic and transcriptomic heterogeneity. Mod Pathol. 2015; 28:340-351.

[9] Lehmann BD, Bauer JA, Chen X, Sanders ME, Chakravarthy $A B$, Shyr $Y$, et al. Identification of human triplenegative breast cancer subtypes and preclinicalmodels for selection of targeted therapies. J Clin Invest. 2011; 121:2750-67.

[10] Lehmann BD, Pietenpol JA. Identification and use of biomarkers in treatment strategies for triple-
Conflict of interest: The authors certify that there is no actual or potential conflict of interest in relation to this article.

negative breast cancer subtypes. J Pathol. 2014; 232:142-150.

[11] Burstein MD, Tsimelzon A, Poage GM, Covington KR, Contreras A, Fuqua SA, et al. Comprehensive genomic analysis identifies novel subtypes and targets of triple-negative breast cancer. Clin Cancer Res. 2015; 21:1688-1698.

[12] Jézéquel $P$, Loussouarn $D$, Guérin-Charbonnel C, Campion L, Vanier A, Gouraud W, et al. Geneexpression molecular subtyping of triple-negative breast cancer tumours: importance of immune response. Breast Cancer Res. 2015; 17:43.

[13] Valentin MD, da Silva SD, Privat M, Molecular insights on basal-like breast cancer. Breast Cancer Res Treat. 2012; 134(1):21-30.

[14] Isakoff SJ, Mayer EL, He L, Traina TA, Carey LA, Krag KJ, et al. TBCRC009: A multicenter phase II clinical trial of platinum monotherapy with biomarker assessment in metastatic triple-negative breast cancer. J Clin Oncol 2015; 33: 1902-1909.

[15] Jiang Z, Jones R, Liu JC, Deng T, Robinson T, Chung PE, et al. RB1 and p53 at the crossroad of EMT and triple-negative breast cancer. Cell Cycle. $2011 ; 15 ; 10: 1563-70$.

[16] Pfister K, Pipka JL, Chiang C, Liu Y, Clark RA, Keller R, et al. Todd Stukenberg. Identification of Drivers of Aneuploidy in Breast Tumors Cell Rep. 2018 ; 29; 23: 2758-2769.

[17] Watkins JA, Irshad S, Grigoriadis A. Genomic scars as biomarkers biomarkers of homologous recombination deficiency and drug response in breast and ovarian cancers. Breast Cancer Res 2014; 16:211.

[18] Robson M, Im SA, Senkus E, Xu B, Domchek $\mathrm{SM}$, Masuda $\mathrm{N}$, et al. Olaparib for Metastatic Breast Cancer in Patients with a Germline BRCA Mutation.N Engl J Med. 2017;377:523-533

[19] Turner NC, Telli ML, Rugo HS, Mailliez M, J Ettl J, Grischke E-M, et al. Phase II ABRAZO: Talazoparib in gBRCA1/2 Mutation-Positive MBC.(Abstract 1007). Presented Jul 102017 ASCO Annual Meeting.

[20] Von Minckwitz G, Schneeweiss A, Loibl S, Salat C, Denkert C, Rezai M, Blohmer JU, et al. Neoadjuvant carboplatin in patients with triple-negative and HER2-positive early breast cancer (GeparSixto; GBG 66): a randomised phase 2 trial. Lancet Oncol 2014; 15:747-56. 
[21] Sikov WM, Berry DA, Perou CM, Singh B, Cirrincione CT, Tolaney SM, et al. Impact of the addition of carboplatin and/or bevacizumab to neoadjuvant once-per-week paclitaxel followed by dose-dense doxorubicin and cyclophosphamide on pathologic complete response rates in stage II to III triple-negative breast cancer: CALGB 40603 (Alliance). J Clin Oncol 2015; 33:13-21.

[22] Hu XC, Zhang J, Xu BH, Cai L, Ragaz J, Wang ZH, et al. Cisplatin plus gemcitabine versus paclitaxel plus gemcitabine as first-line therapy for metastatic triple-negative breast cancer (CBCSG006): a randomised, open-label, multicentre, phase 3 trial. Lancet Oncol 2015; 16:436-46.

[23] Yardley DA, Brufsky A, Coleman RE, Conte PF, Cortes J, Glück $S$, et al. Erratum to: 'Phase II/ III weekly nab-paclitaxel plus gemcitabine or carboplatin versus gemcitabine/carboplatin as first-line treatment of patients with metastatic triplenegative breast cancer (the tnAcity study): study protocol for a randomized controlled trial. Trials. 2016 Feb 3; 17:63.

[24] Tutt A, Ellis P, Kilburn L, Cheang M, Bliss J, Dowsett M, Flanagan J. et al. The TNT trial: A randomized phase III trial of carboplatin (C) compared with docetaxel (D) for patients with metastatic or recurrent locally advanced triple negative or BRCA1/2 breast cancer. (Abstract S3-01). Presented at December 11, 2014. SABCS 2014.

[25] Choi JE, Kang SH, Lee SJ and Bae YK. Androgen receptor expression predicts decreased survival in early stage triple-negative breast cancer. Ann Surg Oncol 2015; 22: 82-89.

[26] McGhan LJ, McCullough AE, Protheroe CA, Dueck $A C$, Lee JJ, Nunez-Nateras R, et al. Androgen receptor-positive triple negative breast cancer: a unique breast cancer subtype. Ann Surg Oncol 2014; 21: 361-367.

[27] Gucalp A, Tolaney S, Isakoff SJ, Ingle JN, Liu MC, Carey LA, et al. Phase II trial of bicalutamide in patients with androgen receptor-positive, estrogen receptor-negative metastatic breast cancer. Clin Cancer Res 2013; 19:5505-12.

[28] Traina TA, Miller K, Yardley DA, O'Shaughnessy J, Cortes J, Gradishar W, et al. Results from a phase 2 study of enzalutamide (ENZA), an androgen receptor $(A R)$ inhibitor, in advanced $A R+$ triplenegative breast cancer (TNBC). J Clin Oncol 2015; 33: S1003.

[29] Adams S, Schmid P, Rugo HS, Loi S, Toppmeyer $D$, Awada A, et al.Phase 2 study of pembrolizumab as first-line therapy for PD-L1-positive metastatic triple-negative breast cancer (mTNBC): Preliminary data from KEYNOTE-086 cohort B (Abstract 1008.) Presented June 3, 2017 ASCO Annual Meeting.

[30] Dalgleish AG. Rationale for combining immunotherapy with chemotherapy. Immunotherapy 2015;7:309-16.

[31] KokM, HorlingsHM, Vijver K. Russell N, Adaptive phase II randomized non-comparative trial of nivolumab after induction treatment in triple negative breast cancer: TONIC-trial (Abstract LBA14) Presented Mar 31, 2017 ESMO Annual Meeting.

[32] Schmid P, Adams S, Rugo HS, Schneeweiss A, Barrios $\mathrm{CH}$, et al; Atezolizumab and NabPaclitaxel in Advanced Triple Negative Breast Cancer.N Engl J Med. 2018 ;379:2108-2121.

[33] YamC,ManiSA,MoulderSL.Targeting theMolecular Subtypes of Triple Negative Breast Cancer: Understanding the Diversityto Progress the Field. Oncologist. 2017; 22:1086-1093.

[34] Carey LA, Rugo HS, Marcom PK, Mayer EL, Esteva FJ, Ma CX, et al. TBCRC 001: randomized phase II study of cetuximab in combination with carboplatin in stage IV triple-negative breast cancer. J Clin Oncol 2012; 30:2615-23.

[35] Baselga J, Gómez P, Greil R, Braga S, Climent MA, Wardley AM, et al. Randomized phase II study of the anti-epidermal growth factor receptor monoclonal antibody cetuximab with cisplatin versus cisplatin alone in patients with metastatic triple-negative breast cancer. J Clin Oncol 2013; 31:2586-92.

[36] Cortes J, O'Shaughnessy J, Loesch D, Blum JL, Vahdat LT, Petrakova K, et al; EMBRACE (Eisai Metastatic Breast Cancer Study Assessing Physician's. Eribulin monotherapy versus treatment of physician's choice in patients with metastatic breast cancer (EMBRACE): a phase 3 open-label randomised study.Lancet.2011; 12; 377:914-23.

[37] Berkey CS, Rosner B, Tamimi RM, Willett WC, Hickey $M$, Toriola $A$, et al. Body size from birth through adolescence in relation to risk of benign breast disease in young women. Breast Cancer Res Treat. 2017;162:139-149.

[38] Lehmann BD, Jovanović B, Chen X, Estrada MV, Johnson KN, Shyr $Y$, et al, Refinement of TripleNegative Breast Cancer Molecular Subtypes: Implications for Neoadjuvant Chemotherapy Selection. PLoS One. 2016; 11: e0157368.

[39] Zhu Y, Zhao F, Li Z, Yu J. Current landscape and future directions of biomarkers for predicting responses to immune checkpoint inhibitors. Cancer Manag Res. 2018; 7; 10:2475-2488.

[40] Diéras V, Campone M, Yardley DA, Romieu G, Valero V, Isakoff SJ, et al. Randomized, phase II, 
placebo-controlled trial of onartuzumab and/or bevacizumab in combination with weekly paclitaxel in patients with metastatic triple-negative breast cancer. Ann Oncol 2015; 26: 1904-1910.

[41] Hon JD, Singh B, Sahin A, Du G, Wang J, Wang $\mathrm{VY}$, et al. Breast cancer molecular subtypes: from TNBC to QNBC Am J Cancer Res 2016;6:18641872.

[42] Barton VN, D'Amato NC, Gordon MA. Androgen Receptor Biology in Triple Negative Breast Cancer: a Case for Classification as AR+ or Quadruple Negative Disease. Horm Cancer 2015; 6: 206-213.

[43] Paik S, KimC, Wolmark N. HER2 status and benefit from adjuvant trastuzumab in breast cancer. $\mathrm{N}$ Engl JMed 2008; 358:1409-1411.

[44] Mittendorf EA, Ardavanis A, Symanowski J, Murray JL, Shumway NM, Litton JK, et al. Primary analysis of a prospective, randomized, single-blinded phase II trial evaluating the HER2 peptide AE37 vaccine in breast cancer patients to prevent recurrence. Ann Oncol 2016; 27:1241-1248.

[45] Prat A, Perou CM. Mammary development meets cancer genomics. Nat Med. 2009; 15:842-4. 